\title{
Numerical Study of Space Charge Effects in a Point Cathode Electron Gun: Distribution of Space Charge Density *
}

\author{
Ryo Iiyoshi ${ }^{\dagger}$ \\ Department of Electrical and Electronic Engineering, \\ Aichi Institute of Technology, Toyota, Aichi 470-0392, Japan \\ (Received 28 November 2007; Accepted 29 February 2008; Published 19 March 2008)
}

\begin{abstract}
The space charge effects in a thermionic emission gun using a tungsten point cathode have been studied with numerical method. The method is based on the integral form of Poisson equation, where the space charges are treated with a large number of coaxial charged rings surrounding the cathode. Each ring charge is estimated from many electron trajectories traced in 3D. The distribution of the space charge density around the cathode of the tip radius $0.4 \mu \mathrm{m}$ was calculated for different bias conditions and different cathode temperatures. The space charge effects were examined by comparing these distributions as well as the axial potential and field distributions near the tip. The numerical results show that even at the temperature as high as $3100 \mathrm{~K}$ the cathode can provide the emission without any noticeable space charge effects when the negative bias on the Wehnelt electrode is shifted by several volts from the cutoff. [DOI: 10.1380/ejssnt.2008.91]
\end{abstract}

Keywords: Electron emission; Thermionic emission; Space charge effects; Computer simulations; Field computation

\section{INTRODUCTION}

Electron emission under low electric field produces a space charge cloud in front of the emitter surface. The negative charges reduce the field strength near the surface and limit the emission current density. Thermionic emission guns using a $\mathrm{LaB}_{6}$ cathode or a tungsten hairpin cathode are operated under space-charge-limited conditions. These cathodes have relatively large tip radii of $10-50 \mu \mathrm{m}$. The cathode tip is placed behind the negatively biased Wehnelt electrode. The geometrical arrangement causes low field near the tip and the resultant space charges limit the emission current density and thus the beam brightness of the gun. This limitation can be removed by the use of a point cathode having a sharply etched tip of submicron radii. The small tip produces accelerating field higher than the large one. In this case, the space charges become important at higher cathode temperatures or higher emission densities. The study of such effects in practical electrode geometry requires the numerical determination of the field, because the distribution of the space charges is unknown prior to a rigorous self-consistent calculation of Poisson equation [1].

A numerical method has been developed to study the space charge effects in a thermionic emission gun with a tungsten point cathode [2]. This method is based on the integral form of Poisson equation, where the space charges around the cathode are approximated by a large number of the coaxial charged rings. The method has an advantage that the cathode tip in submicron size and the other electrodes with large dimensions can be easily considered with accuracy in the field determination, because the electrode geometry is treated in the same manner as the charge density method (CDM) or the surface charge method (SCM). It had been described in a previous paper [3] that the method gives the emission cur-

* This paper was presented at 6th International Symposium on Atomic Level Characterizations for New Materials and Devices, Kanazawa, Japan, 28 October-2 November, 2007.

†Corresponding author: iiyoshi@aitech.ac.jp rents near the measured values, when the space charges are estimated from the rays traced with random emitting conditions having the energy and angular distributions of thermionic emission.

This paper describes the distribution of the space charge density at different operating conditions. Using the method, the space charge density around the cathode was examined for different bias conditions and different cathode temperatures. The distribution was visualized as gray scale maps. They show the variation of the space charge distribution caused by the adjustments of the bias and the cathode temperature of the gun. After a brief description of the method, the numerical results are represented. The space charge effects in the gun are discussed by comparing some of the distributions and the potential and field distributions along the optical axis.

\section{METHODS}

\section{A. Numerical model of the gun}

The electrode configuration of the gun is shown in Fig. 1. The gun consists of the point cathode, the Wehnelt electrode and the anode. The cathode is a tungsten wire of $0.1 \mathrm{~mm}$ in diameter. It has the spherical tip of $0.4 \mu \mathrm{m}$ in radius of curvature. The half angle of the cone part near the tip is about $8^{\circ}$. The cathode tip is placed at a height of $0.25 \mathrm{~mm}$ from the Wehnelt aperture. The aperture size is $1.2 \mathrm{~mm}$ in diameter and $0.1 \mathrm{~mm}$ in thickness. The anode is placed at a distance of $9.2 \mathrm{~mm}$ from the cathode tip. The anode has the opening of $4 \mathrm{~mm}$ in diameter.

The surface of each electrode was divided into small sections for the numerical determination of the surface charge density. The surface charge density is closely related to the surface field on each section and it largely varies at the tip part of the cathode and at the corners of the Wehnelt aperture and the anode opening. So these parts were divided into smaller sections to keep an accuracy of the field determination. The total number of the surface sections was 200 in this study. 


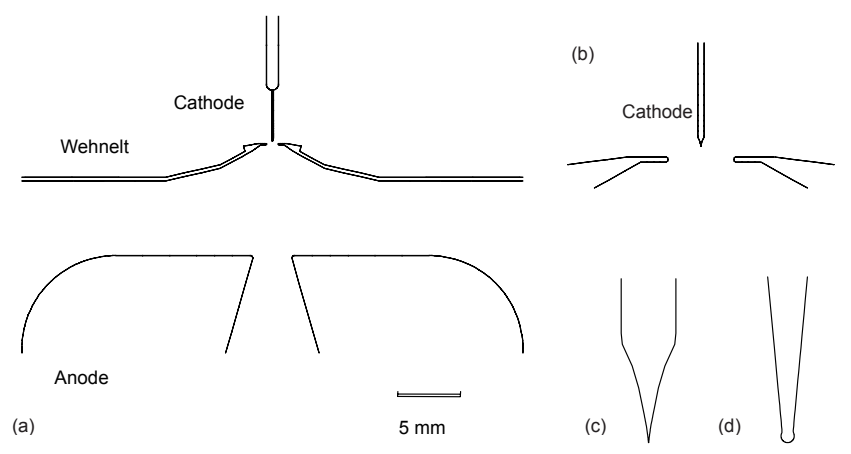

FIG. 1: Numerical model of the electron gun. (a) Triode system. (b) Enlarged view near the Wehnelt aperture. The cathode tip is placed at a height of $0.25 \mathrm{~mm}$ from the aperture of $1.2 \mathrm{~mm}$ in diameter. (c) and (d) Enlarged views of the cathode cone and tip. The tip radius is $0.4 \mu \mathrm{m}$.

\section{B. Field determination}

\section{Iterative procedure}

The integral form of Poisson equation was solved at the anode voltage of $50 \mathrm{kV}$. The field including the space charges was determined by the iteration procedure, consisting of (i) the field determination, (ii) the direct raytracing in 3D, and (iii) the estimation of the space charges from the traced rays. First the field was obtained in the same way as the CDM. The CDM gives the surface charge densities on the electrodes in the case of no space charge. So the electron trajectories were traced in the field, and then the space charges were estimated from the trajectories. The space charges were treated as a large number of coaxial charged rings. Since the space charges cause negative potential, the surface charge densities were recalculated considering the space charge potential on each section of the electrode surfaces. The recalculation led to the renewed field distribution, so that the trajectories were traced again in the field to obtain the renewed distribution of the space charge density. The self-consistent result of Poisson equation was obtained by the repetition of these calculations.

\section{Ray tracing}

The electron trajectories were traced in $3 \mathrm{D}$ by solving directly the equation of motion. The electrons were started from a relatively large area of the cathode including the negatively biased area. The cathode surface from $z=0$ (the tip apex) to $z=-35 \mu \mathrm{m}$ (or $-50 \mu \mathrm{m}$ ) were divided into 184 (or 259) circular sections with different widths of $\Delta z$, and then fifty rays were started from each section. The number of the traced rays amount to 9,200 (or 12,950). The temperature rise of the cathode causes the energy broadening of the emitting electrons. This effect was taken into account in the ray tracing. The emitting energies were given by random numbers having the Maxwell-Boltzmann distribution. The emitting angles were also given by random numbers having the angular distributions of thermionic emission, where the azimuths were provided by quasi-uniform random numbers [3].

\section{Estimation of the space charges}

The space around the cathode was divided into a small volume element $\Delta V=\pi r \delta r \delta z(\delta r=\delta z=0.2 \mu \mathrm{m})$. The space charge $\Delta Q$ in each volume element was estimated from the rays passing through it as follows;

$$
\Delta Q=\sum_{n} i_{n} \Delta \tau_{n}
$$

where $n$ is the total number of the rays, $i_{n}$ and $\tau_{n}$ are the current and the transit time of each ray. The space charge density is given by

$$
\Delta \rho=\Delta Q / \Delta V
$$

The current $i_{n}$ of each ray was determined from the emission current $\Delta I$ of each circular section $\Delta z$. The current $\Delta I$ was calculated using the Richardson-Dushman equation,

$$
\Delta I=J \Delta S=A T^{2} \exp \left(\frac{-\phi}{k T}\right) \Delta S,
$$

where $A$ is the Richardson constant, $T$ the cathode temperature, $\phi$ the work function, $k$ the Boltzmann constant, and $\Delta S$ the area of each circular section $\Delta z$. In the case that there is the accelerating field in front of the cathode surface, the emission current was calculated by adding the Schottky factor $f_{\text {sch }}$;

$$
\begin{aligned}
\Delta I & =J f_{\text {sch }} \Delta S \\
& =A T^{2} \exp \left(\frac{-\phi}{k T}\right) \exp \left(\frac{0.4404 \sqrt{|F|}}{T}\right) \Delta S,
\end{aligned}
$$

where $|F|$ is the field strength on the cathode surface. The calculated current $\Delta I$ was equally assigned to fifty rays from the area, $i=\Delta I / 50$. The Richardson constant and the work function were put to $A=80 \mathrm{~A} / \mathrm{cm}^{2} \mathrm{~K}^{2}$ and $\phi=4.5 \mathrm{eV}$, which give the current density $J$ of $5.0 \mathrm{~A} / \mathrm{cm}^{2}$ at $2800 \mathrm{~K}, 14.3 \mathrm{~A} / \mathrm{cm}^{2}$ at $2950 \mathrm{~K}$ and $37.1 \mathrm{~A} / \mathrm{cm}^{2}$ at $3100 \mathrm{~K}$.

\section{RESULTS AND DISCUSSION}

The iteration was continued as monitoring some of the intermediate results, such as the potential distribution and electron trajectories near the cathode as well as the anode current or the emission current. The emission current was obtained by adding the currents assigned to the traced rays passing through the Wehnelt aperture. The iterative method provided a stable value of the emission current after several iterations, where we obtained the self-consistent result of the field. One of the measures for the validity of the field determination is that the iteration leads to the emission currents corresponding to the measured values at different biases and cathode temperatures. Figure 2 shows the calculated emission currents as a function of the Wehnelt bias voltage. The cutoff bias is about $-220 \mathrm{~V}$. The current is increased as reducing the negative bias voltage. At $3100 \mathrm{~K}$ the iteration provides the emission current of about $120 \mu \mathrm{A}$ at the bias $-198 \mathrm{~V}$. 


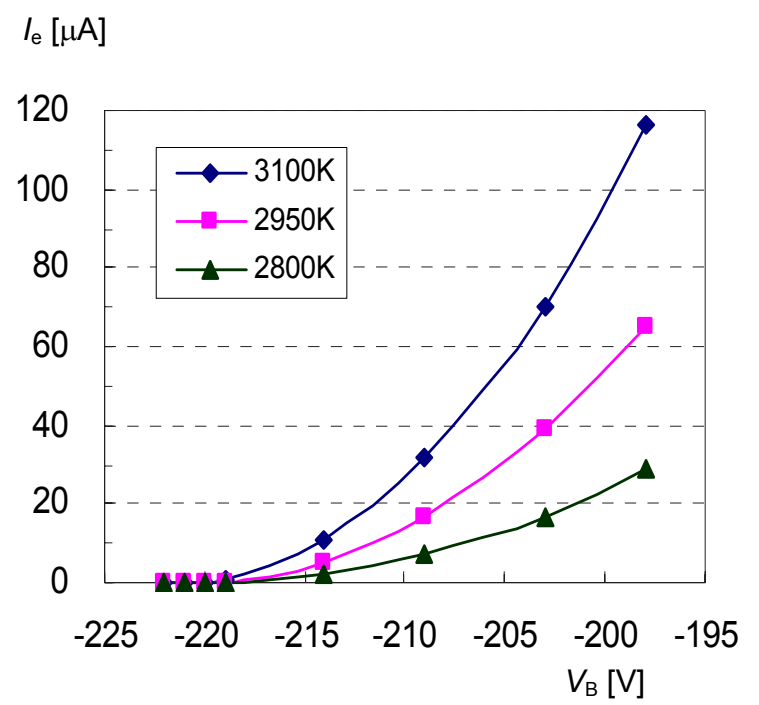

FIG. 2: Calculated emission current $I_{e}$ as a function of the Wehnelt bias $V_{B}$ at the cathode temperature of $2800 \mathrm{~K}, 2950 \mathrm{~K}$ and $3100 \mathrm{~K}$. Anode voltage $50 \mathrm{kV}$.

The current was over $270 \mu \mathrm{A}$ in the case of Laplace equation. The space charges restrict the emission currents to low values at the cathode temperatures above $2800 \mathrm{~K}$. The numerical method provides the emission currents near the measured values $[3,4]$.

The distribution of the space charge density was estimated from the traced rays. Figure 3 shows the distributions at the Wehnelt bias of $-214 \mathrm{~V}$; (a) the cathode temperature $2800 \mathrm{~K}$, (b) $2950 \mathrm{~K}$ and (c) $3100 \mathrm{~K}$. The bias is the value shifted by $6 \mathrm{~V}$ from the cutoff $(-220$ $\mathrm{V})$. The gray scale maps correspond to the area of $z$ from $-40 \mu \mathrm{m}$ to $10 \mu \mathrm{m}$ and $r \leq 22 \mu \mathrm{m}$ (the tip apex of the cathode at $z=0, r=0)$. The maps include the coaxial charged rings of 11,680-11,960. It can be seen that the space charge densities are increased with the cathode temperature or the emission current density. The cone part is surrounded with a dense space charge cloud, which is caused by the electrons repelled with the negative potentials. These electrons have low velocities or long transit time and contribute twice to the space charge density, once before the repelling and another after it. The density becomes low at the tip part. The electrons from the tip cause a thin layer of the space charges, which means that they are accelerated within a short distance. The accelerating field produces a low density area of the space charges along the optical axis in front of the cathode tip.

The bias adjustment varies the field distribution around the cathode, which causes the variation of the space charge distribution. Figure 4 shows the distributions of the space charge density when the bias is reduced to $-209 \mathrm{~V}$ (the bias shift is $11 \mathrm{~V}$ from the cutoff). The number of the charged rings becomes 19,730-20,370 at this bias. The dense area is moved upward and the radial distribution is increased as compared with Fig. 3. The comparison also shows that the bias reduction is effective in diminishing the space charge density in front of the tip. The low density area around the optical axis is enlarged when the bias is reduced.

Typical values of the space charge density at the bias $-214 \mathrm{~V}$ and $-209 \mathrm{~V}$ are listed in Table I. The density

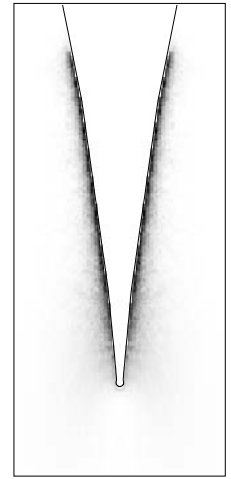

(a)

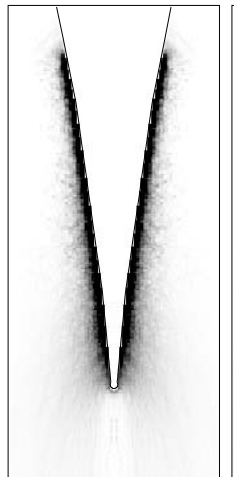

(b)

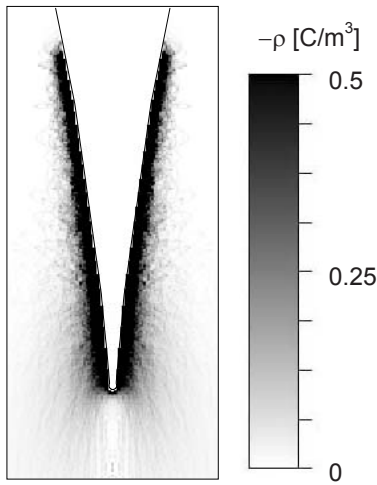

(c)
FIG. 3: Distribution of the space charge density at the bias voltage of $-214 \mathrm{~V}$. (a) Cathode temperature $2800 \mathrm{~K}$, (b) $2950 \mathrm{~K}$ and (c) $3100 \mathrm{~K}$. The area corresponds to $-40 \mu \mathrm{m}$ $\leq z \leq 10 \mu \mathrm{m}, r \leq 22 \mu \mathrm{m}$. The bias is a value shifted by $6 \mathrm{~V}$ from the cutoff.

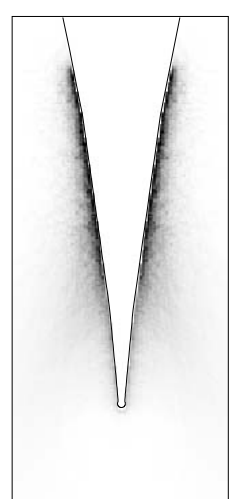

(a)

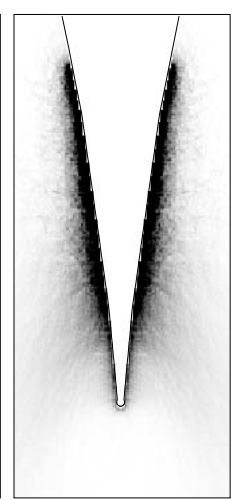

(b)

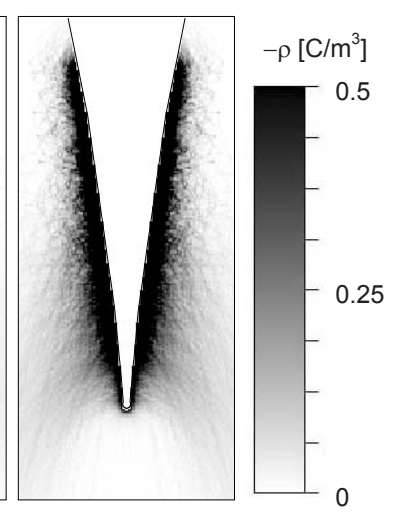

(c)
FIG. 4: Distribution of the space charge density at the bias voltage of $-209 \mathrm{~V}$. (a) Cathode temperature $2800 \mathrm{~K}$, (b) $2950 \mathrm{~K}$ and (c) $3100 \mathrm{~K}$. The bias is the value shifted by $11 \mathrm{~V}$ from the cutoff.

$\rho_{\text {tip }}$ is the value in the vicinity of the cathode tip and the density $\rho_{\max }$ the maximum value at the cone part near $z=-35 \mu \mathrm{m}$. Both values are increased when the cathode temperature is raised. At each temperature the density $\rho_{\text {tip }}$ has a value of one order magnitude smaller than $\rho_{\max }$. The density $\rho_{\text {tip }}$ causes a decrease in the surface field of the cathode tip, which becomes smaller at the bias $-209 \mathrm{~V}$. The bias shift has no large influence on the maximum density $\rho_{\max }$ at each temperature. The space charges around the cone part cause more negative potentials than those near the tip and reduce the emission area [3].

The space charge effects were examined by comparing

TABLE I: Space charge density $\rho_{\text {tip }}$ in front of the cathode tip and the maximum value $\rho_{\max }$ at the cone part. The bias $V_{B}=-214 \mathrm{~V}$ and $-209 \mathrm{~V}$ (the cutoff bias $-220 \mathrm{~V}$ ).

\begin{tabular}{ccccc}
\hline \hline & \multicolumn{2}{c}{$V_{B}=-214 \mathrm{~V}$} & \multicolumn{2}{c}{$V_{B}=-209 \mathrm{~V}$} \\
$T[\mathrm{~K}]$ & $\rho_{\text {tip }}\left[\mathrm{C} / \mathrm{m}^{3}\right]$ & $\rho_{\max }\left[\mathrm{C} / \mathrm{m}^{3}\right]$ & $\rho_{\text {tip }}\left[\mathrm{C} / \mathrm{m}^{3}\right]$ & $\rho_{\max }\left[\mathrm{C} / \mathrm{m}^{3}\right]$ \\
\hline 2800 & -0.062 & -0.558 & -0.051 & -0.612 \\
2950 & -0.180 & -1.527 & -0.140 & -1.582 \\
3100 & -0.676 & -3.898 & -0.407 & -3.878 \\
\hline \hline
\end{tabular}




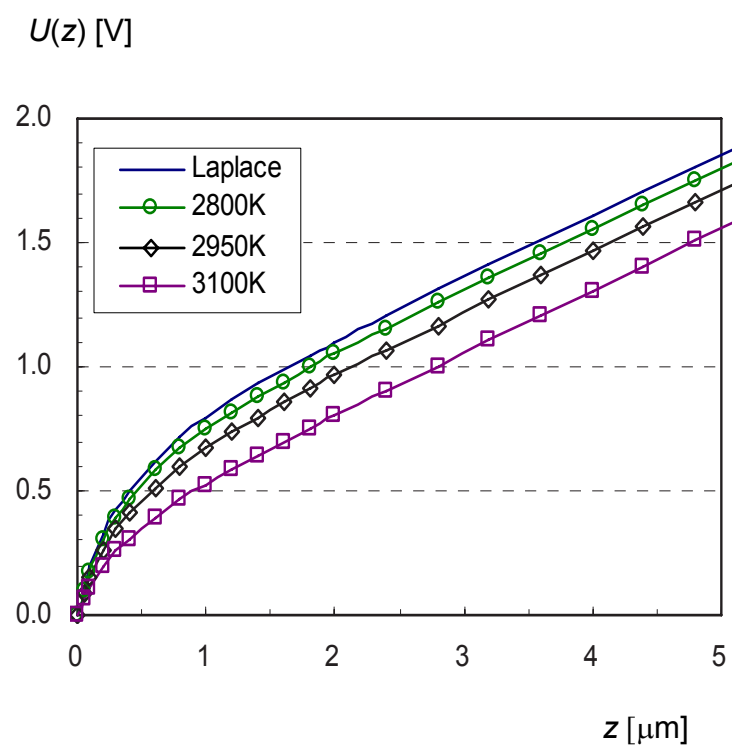

FIG. 5: Axial potential distribution $U(z)$ at the bias voltage of $-214 \mathrm{~V} . U(z)$ in $V$. The potential is decreased by the space charge effects when the cathode temperature is raised. The solution of Laplace equation is also given for comparison.

the potential and field distributions along the optical axis. The potential $U(z)$ and the field strength $|F(z)|$ at the bias $-214 \mathrm{~V}$ are given in Figs. 5 and 6 , where the corresponding values before iteration (a solution of Laplace equation) are also given for comparison. These values were obtained by the numerical integration of the surface charge densities on the electrodes and the space charge densities around the cathode $[2,3]$. At $2800 \mathrm{~K}$ the potential and the field have nearly the same values as in the case of Laplace equation, which means that the space charge effects are small at this temperature. The differences of the potential and the field distributions become large when the temperature is raised to $2950 \mathrm{~K}$ and $3100 \mathrm{~K}$.

The field strength is maximized at the tip surface and it is decreased within a narrow area near the tip as shown in Fig. 6. This is the characteristic of the point cathode in the triode gun with the negatively biased Wehnelt electrode. The field strength at the tip $(z=0)$ is of the order of $10^{6} \mathrm{~V} / \mathrm{m}$. Using a planar diode model of a small spacing and the Child-Langmuir equation, we can evaluate the anode voltage giving the emission current density equivalent to the Richardson-Dushman equation. At the anode voltage, the diode gives the space-charge-free emission. So we obtain the minimum field strength for the spacecharge-free emission from the anode voltage and the diode spacing [5]. The evaluation leads to the minimum field strength of $2.2 \times 10^{5}-2.9 \times 10^{5} \mathrm{~V} / \mathrm{m}$ for the diode spacing of $0.4-1 \mu \mathrm{m}$. The field strengths in Fig. 6 are greater than the evaluated values. This means that the bias shift of $6 \mathrm{~V}$ from the cutoff allows the space-charge-free emission from the tip even at $3100 \mathrm{~K}$, when the gun is operated with the cathode having the tip radius of $0.4 \mu \mathrm{m}$ at given electrode geometry shown in Fig. 1. The field strength $|F(0)|$ gives the Schottky factor of the order of unity.

Experiments showed that the beam brightness of the point cathode gun is maximized at the bias shift of several

\section{$|F(z)|$}

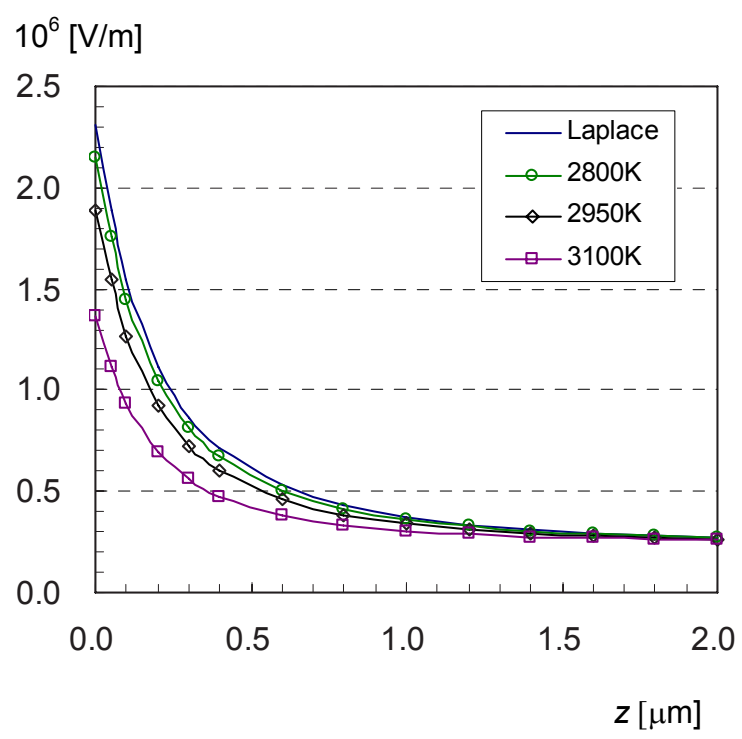

FIG. 6: Axial field distribution $|F(z)|$ at the bias voltage of $-214 \mathrm{~V} .|F(z)|$ in $\mathrm{V} / \mathrm{m}$. The cathode tip of $0.4 \mu \mathrm{m}$ in radius produces high field strength near the tip.

volts from the cutoff $[6,7]$. This bias is called the optimum bias, where the gun provides the solid Gaussian beam with the brightness near the Langmuir's theoretical value. At the optimum bias, the total beam current is limited to a value of the order of $10 \mu \mathrm{A}$, which corresponds to the calculated emission currents shown in Fig. 2. In the bias range of less than the optimum, the beam current and thus the brightness are reduced by the space-chargelimitation on the emission. Numerical results described above indicate that the method can be utilized for the quantitative estimation of the space charge effects in the point cathode gun.

\section{CONCLUSIONS}

The distribution of the space charge density in the point cathode gun has been studied with numerical method. To determine the field including the space charges, the integral form of Poisson equation was solved by the iterative procedure, where a large number of the electron trajectories were traced with random emitting energies and angles. The space charge density around the cathode was estimated at different bias conditions and different cathode temperatures. The distributions were given in gray scale maps, which made clear the variation of the space charge density caused by the adjustments of the bias and the cathode temperature. The space charge effects were examined by comparing the axial potential and field distributions near the cathode. The results show that the space charge effects in front of the cathode tip become small at the bias shift of several volts from the cutoff, even when the gun is operated at the cathode temperature as high as $3100 \mathrm{~K}$ for the given electrode configurations. The method provides the numerical results that agree with the practical gun operation at high cathode temperatures. 
[1] P. W. Hawkes and E. Kasper, Principles of Electron Optics, Vol. 2, p. 953 (Academic Press, London, 1989).

[2] R. Iiyoshi, Nucl. Instrum. Meth. Phys. Res. A519, 297 (2004).

[3] R. Iiyoshi, e-J Surf. Sci. Nanotech. 4, 339 (2006).

[4] R. Iiyoshi, H. Takematsu, and S. Maruse, J. Electron Microsc. 37, 1 (1988).
[5] A. Ohshita, H. Shimoyama, and S. Maruse, J. Electron Microsc. 27, 253 (1973)

[6] R. Iiyoshi and S. Maruse, J. Electron Microsc. 45, 128 (1996).

[7] R. Lauer, Advances in Optical and Electron Microscopy, vol. 8, p. 137 (Academic Press, London, 1982). 